\title{
Alterstice
}

Revue internationale de la recherche interculturelle

International Journal of Intercultural Research

Revista International de la Investigacion Intercultural

\section{Penser la diversité par les transitions de la santé mentale}

\section{Ilario Rossi}

Volume 4, numéro 2, 2014

Santé mentale et sociétés plurielles

URI : https://id.erudit.org/iderudit/1077424ar

DOI : https://doi.org/10.7202/1077424ar

Aller au sommaire du numéro

\section{Éditeur(s)}

Alterstice

ISSN

1923-919X (numérique)

Découvrir la revue

Citer cet article

Rossi, I. (2014). Penser la diversité par les transitions de la santé mentale. Alterstice, 4(2), 39-48. https://doi.org/10.7202/1077424ar

\section{Résumé de l'article}

L'émergence d'une culture de la mobilité - notamment des personnes, des savoirs, des pratiques, des techniques - s'accompagne d'une valorisation progressive de la diversité. Cette mouvance sociétale pose de nouveaux défis institutionnels et professionnels, notamment en santé mentale. Parallèlement, ce domaine est traversé par des injonctions politiques, économiques et culturelles importantes qui modifient à leur tour l'organisation des pratiques cliniques. Pour esquisser des pistes de réflexion, cet article propose de mettre en lien la diversité du sujet dans la clinique avec un changement de culture de la pratique. Comment le jaillissement de la diversité coexiste-t-il avec ces transitions ? Quelles perspectives interprétatives se dessinent-elles ? Pour cerner le contexte à partir duquel se pense la diversité en santé mentale, nous nous concentrerons sur les changements économiques et les réformes politiques, le statut des professionnels et les missions de la clinique, l'émergence du sujet et la valorisation de l'autonomie. En ce sens, s'esquisse un système à partir duquel le soignant raisonne et interagit avec la diversité. En valorisant la perspective synthétique davantage que la focale détaillée, en utilisant des sources théoriques au détriment de données empiriques, la diversité se donne à lire comme la résultante d'un ensemble organisé, de ses multiples agencements et de ses logiques d'influence. Selon cette perspective, elle se définit moins comme un objet institué que comme une composante d'un processus instituant, défini par les injonctions multiples qui traversent la clinique contemporaine. 


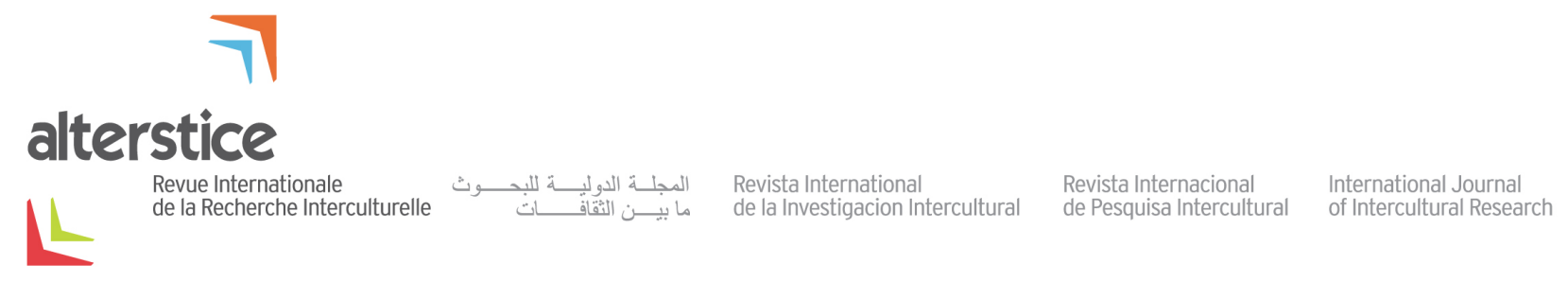

ARTICLE THÉMATIQUE

\section{Penser la diversité par les transitions de la santé mentale}

Ilario Rossi ${ }^{1}$

\section{Résumé}

L'émergence d'une culture de la mobilité - notamment des personnes, des savoirs, des pratiques, des techniques s'accompagne d'une valorisation progressive de la diversité. Cette mouvance sociétale pose de nouveaux défis institutionnels et professionnels, notamment en santé mentale. Parallèlement, ce domaine est traversé par des injonctions politiques, économiques et culturelles importantes qui modifient à leur tour l'organisation des pratiques cliniques. Pour esquisser des pistes de réflexion, cet article propose de mettre en lien la diversité du sujet dans la clinique avec un changement de culture de la pratique. Comment le jaillissement de la diversité coexiste-t-il avec ces transitions? Quelles perspectives interprétatives se dessinent-elles? Pour cerner le contexte à partir duquel se pense la diversité en santé mentale, nous nous concentrerons sur les changements économiques et les réformes politiques, le statut des professionnels et les missions de la clinique, l'émergence du sujet et la valorisation de l'autonomie. En ce sens, s'esquisse un système à partir duquel le soignant raisonne et interagit avec la diversité. En valorisant la perspective synthétique davantage que la focale détaillée, en utilisant des sources théoriques au détriment de données empiriques, la diversité se donne à lire comme la résultante d'un ensemble organisé, de ses multiples agencements et de ses logiques d'influence. Selon cette perspective, elle se définit moins comme un objet institué que comme une composante d'un processus instituant, défini par les injonctions multiples qui traversent la clinique contemporaine.

\section{Rattachement de l'auteur}

${ }^{1}$ Institut des sciences sociales, Université de Lausanne, Suisse

\section{Correspondance}

ilario.rossi@unil.ch

\section{Mots clés}

diversité, santé mentale, politiques de santé, systèmes de santé, sujet, professionnel de la santé

\section{Pour citer cet article}

Rossi, I. (2014). Penser la diversité par les transitions de la santé mentale. Alterstice, 4(2), 39-x. 


\section{Introduction}

Le $21^{\mathrm{e}}$ siècle se déploie dans un contexte de grandes transformations : l'émergence simultanée de diverses formes de mondialisation - notamment la mobilité des personnes, des techniques, des savoirs et des pratiques, l'internationalisation des marchés économiques et financiers, la révolution numérique et les innovations technologiques - participe à un processus qui entraîne des mutations culturelles inédites et fondamentales (Rossi, 2012). Chacune d'entre elles est à la fois spécifique et diversifiée et, en même temps, amarrée aux autres dans une relation d'influences et d'interdépendances réciproques. Ces processus forment un système et il serait inapproprié de les considérer aujourd'hui séparément.

Nous devons donc apprendre à les penser ensemble, notamment pour saisir les transformations qui modifient actuellement le domaine de la santé mentale et, plus particulièrement, leurs effets sur l'organisation des institutions et la finalité des pratiques professionnelles. C'est donc à travers une lecture systémique de ces transitions que la notion de diversité sera ici contextualisée. Pour éclairer une telle perspective, l'attention sera dirigée dans un premier temps vers les changements sémantiques de cette notion et les enjeux interactionnels qui en découlent au niveau de la relation de soin. Dans un deuxième temps, l'articulation de plusieurs registres politiques et systèmes de santé, cultures institutionnelles, statut des professionnels - permettra d'entrevoir les logiques par lesquelles le domaine de la santé publique et, plus spécifiquement, celui de la santé mentale construit le projet clinique, ses objectifs et ses modalités. La conclusion propose une mise en perspective de la diversité à l'aune des transitions dans le domaine de la santé mentale, en montrant comment le lieu de leur convergence réelle, la clinique, est soumis à des injonctions contradictoires et paradoxales. Le regard proposé, qui ne peut être que général et approximatif propose, en assumant cette posture, une lecture susceptible de repositionner l'émergence d'une clinique de la diversité et de montrer ses potentielles dérives et instrumentalisations.

\section{Émergence de la diversité}

La notion de culture constitue depuis longtemps un centre d'intérêt scientifique et théorique dans le domaine de la santé mentale (Cognet et Montgomery, 2007; Domenig, 2007; Goguikian Ratcliff et Strasser, 2009; Sabatier et Douville, 2002; Tison, 2007). Nombre d'auteurs, de recherches et de publications ont contribué à son émergence en psychiatrie, à l'institutionnalisation d'une clinique intégrant les différences culturelles, au développement de pédagogies valorisant les compétences inter- et transculturelles. Toutes ces perspectives conceptuelles reposent essentiellement sur une vision centrée sur l'individu, son origine et ses trajectoires de migration, ses spécificités d'appartenances sociales, religieuses et communautaires tout en adaptant les procédés interactionnels, communicationnels et relationnels. Par le prisme d'un regard sur l'autre, s'est établie une riche production scientifique, plurielle et variable selon les courants et les régions géographiques de sa réalisation. Cette production a notamment traité des rapports entre psychisme et culture, entre identité et altérité, entre processus migratoires et politiques d'immigration, entre multiculturalisme et interculturalité. Force est de constater que les écarts sémantiques, et les conséquences politiques, de l'usage de la notion de culture traduisent la ferveur des débats scientifiques et des enjeux cliniques (Sabatier et Douville, 2002). Il suffit de se référer aux critiques, parfois virulentes, qui ont accompagné l'essor des acceptions d'une part culturalistes - « une culture fonctionnelle assure la clôture du groupe et lui donne une conscience de son identité " (Nathan, 1994, p. 213) - et d'autre part universalistes - pour lesquelles la culture constitue une variable exogène résiduelle. Dans le domaine de la psychiatrie par exemple, les tenants de la position universaliste dominante s'appuient sur des outils diagnostiques élaborés en Occident, tels que le Diagnostic and Statistical Manual of Mental Disorders (DSM), dont la pertinence théorique et pratique a été contestée, notamment par nombreux anthropologues et philosophes (Kleinmann, 1995; Littlewood, 2002; Demazeux, 2013). Ils remettent en question la légitimité d'appliquer des concepts généraux et des catégories standardisées, produits dans un espace et un temps spécifiques, à des individus provenant de sociétés où ils ne sont pas applicables (Selod, 2001). Aujourd'hui, ces oppositions se reconfigurent et se complexifient : au sein du processus de mondialisation, et pour la première fois dans l'Histoire, "les personnes, les communautés, les cultures et les populations ont une destinée commune " (Garcìa Canclini, 2011, p. 106). Un nouveau regard se façonne, en adéquation avec cette nouvelle réalité : on passe d'une sensibilité multiculturelle - respect des différences et valorisation de l'interculturel - à une posture transculturelle, qui implique une vision dynamique et processuelle de la culture et de ses expressions. II en découle la nécessité de se 
distancier radicalement d'un essentialisme culturel et d'une conception fermée de la culture, qui implique une pression d'homogénéisation interne et de délimitation externe. Au contraire, avec la mondialisation, s'accentue le caractère variable des cultures contemporaines ainsi que leur mouvement incessant de réticulation externe et d'hybridation interne (Welsch, 2005).

Ce glissement sémantique n'est pas anodin. Plus particulièrement, la potentialité transnationale des individus, qui se définit par la circulation entre cultures et modes de vie, promeut la dynamique transculturelle des sociétés auxquelles ils appartiennent, et réciproquement (Welsch, 2005). Cette réciprocité conduit à redéfinir le concept de diversité, cette dernière devenant consubstantielle à toute production d'identité, entre construction de soi et reconnaissance d'autrui, entre transculturalité individuelle et transculturalité sociale : il est désormais nécessaire de penser les identités culturelles comme n'étant pas définies une fois pour toutes mais comme étant toujours en formation, se redéfinissant en permanence sous la pression, en particulier, d'apports culturels extérieurs. Selon cette perspective, la notion de différence culturelle en tant qu'entité permanente et établie s'efface au profit d'une diversité humaine, processuelle et dynamique. De fait, évoquer la diversité ne renvoie plus ni à des questions de territorialité, ni à des prérogatives de nationalité ou d’ethnie mais de "laicité de tolérance » (Wolton, 2008) : elle engage désormais les valeurs intrinsèques aux droits de l'Homme et repose sur une idéologie universelle promouvant la possibilité pour tout un chacun de se construire et de se penser dans sa singularité et dans sa diversité dans un contexte de pluralité.

L'émergence de la diversité comme événement de la singularité accompagne le passage d'une société marquée par la référence à la discipline, au conflit et à la culpabilité à une société qui valorise davantage l'autonomie, la responsabilité et la libre initiative. L'individu contemporain devient autonome, maître de ses choix et de la capacité d'évoluer et de se construire, responsable de lui-même, de ses activités, de ses succès, mais aussi de ses échecs et de ses défaites (Ehrenberg et Lovell, 2005; Ehrenberg, 2010). En ce sens, tout cheminement individuel s'inscrit désormais au cœur d'un destin collectif; il donne naissance à une configuration sociale et culturelle complexe que nous appelons le sujet : devenir sujet se traduit par la volonté de l'individu d'être l'acteur de sa propre existence, par l'intensité par laquelle il mène sa quête de soi et lui confère une importance centrale (Touraine, 2005). Entre l'être humain et l'être de la société, le sujet advient comme élément de médiation face à l'ensemble des déterminations plus ou moins contradictoires qui le constituent, il traduit le processus par lequel il se construit luimême à partir d'un déjà là (de Gaulejac, 2009). Plus l'identité est centrale, plus le corps est important (Rossi, 2013). Notre corps est l'une des évidences de notre existence, puisque c'est dans et avec notre corps que nous sommes nés, que nous vivons, que nous mourrons et dans et avec notre corps que nous construisons nos relations à autrui et notre singularité (Marzano, 2007, p. 7). Par son corps, le sujet concrétise sa diversité, parce que tout au long de la vie, la quête de soi influence, voire transforme, la structure d'ensemble de notre expérience existentielle, morale et corporelle, ainsi que nos jugements et nos actions. C'est l'un des sens du concept de subjectivation, par lequel les dynamiques d'élaboration du rapport à soi, aux autres et au monde se construisent dans l'expérience du corps. La diversité nous parle moins d'une culture que d'une subjectivité et d'une corporéité spécifiques.

Il en découle une vision de la santé individuelle adaptée, s'éloignant d'un état pour devenir elle aussi un processus et se constituer comme «le résultat d'une interaction de facteurs biologiques, psychologiques [...], sociaux, cognitifs et affectifs ", qui s'influencent mutuellement tout le long d'une trajectoire de vie (Sabatier et Douville, 2002 , p. 7). Cette représentation de la santé, ajustée aux transformations de l'individu et capable en même temps de le situer, s'ouvre aux spécificités de la singularité et de la diversité. Elle sollicite, dans toute réponse soignante, une relation de soin centrée sur la personne, ouverte à ses spécificités, à la narrativité de son parcours biographique, de ses appartenances familiales, communautaires et sociales. Une relation de nuances et d'adaptabilité interactionnelles, qui puisse garantir le respect de la personne dans sa diversité et les modalités cliniques capable de la reconnaître (Goguikian Ratcliff et Strasser, 2009). Cependant, cette relation de soin ne se réduit pas à elle-même : perméable aux influences contextuelles dans lesquelles elle se déploie, elle s'ajuste aux conditions structurelles qui lui permettent de se mettre en scène. 


\section{Les transformations structurelles de la santé}

Si l'expérience de la maladie relève de l'individuel, la santé, elle, participe aussi du collectif et se définit désormais comme une construction socialement et politiquement élaborée dont les limites sont constamment rediscutées. Domaine multidimensionnel aux interactions complexes, elle constitue, dans une perspective anthropologique, un moyen d'accès au système des interprétations et des valeurs d'une société. Elle est ainsi un révélateur du social, notamment dans l'expression des facteurs qui la constituent - l'économique, le politique et le scientifique -, qui la façonnent et la caractérisent selon les contextes.

En particulier, avec la mondialisation de la production et des échanges, ainsi que son corollaire, la séparation entre économie d'une part et social et politique de l'autre, la médecine a intégré structurellement l'exigence de rentabilité et la règle de la compétitivité comme opérateurs du marché de la santé (Gori et Del Volgo, 2008; Rossi, 2002). Une nouvelle configuration se dessine, reposant sur l'exigence de relier planification et évaluation, en promouvant par des décisions politiques une culture réformiste des systèmes de santé. De fait, les pays européens et leurs gouvernements ont entrepris de réformer leurs systèmes de santé, dans le but de promouvoir un changement structurel passant par le réexamen des actions et des stratégies économiques en matière de soins. Les programmes des réformes se sont traduits par l'application progressive à l'ensemble des institutions de soins d'un nouveau mode d'organisation - le management care et le management disease -, conduisant à réglementer plus strictement les comportements des principaux acteurs des secteurs de la santé (Castro et Singer, 2004). En même temps, ces mêmes secteurs sont soumis à la restructuration et à la concurrence. Il en découle que toute pratique clinique est indissociable des réponses qu'elle est censée fournir: la recherche d'efficacité thérapeutique s'accompagne d'une nouvelle exigence, celle de l'efficience de la pratique soignante et médicale, pour laquelle chaque stratégie d'intervention se voit aussi assigner une valeur financière. Les objectifs sont explicites: rationaliser et coordonner les prestations pour mieux contrôler les dépenses, et créer de nouvelles références en matière de droits et de devoirs pour les professionnels et les patients. Cependant, la quête d'efficience doit aussi respecter, sous l'impulsion du politique, un certain nombre de conditions, portant notamment sur des critères comme la satisfaction des besoins, l'accessibilité, la solidarité, l'équité, le respect d'une éthique de la pratique, la déontologie - sans lesquelles il serait contradictoire de conjuguer médecine, soins, économie et politique.

Parallèlement, les innovations technologiques influencent la relation au malade et l'activité des professionnels. Tout individu devient non seulement prévisible, mais surtout maîtrisable par la biologie de ses constituants et la prescription de médicaments. Les questions qui en découlent touchent désormais à ce que nous sommes en tant qu'êtres humains et non plus seulement à ce que nous pouvons faire comme êtres humains (Kehr, Niewöhner et Vailly, 2011). D'autre part, I'introduction progressive des nouvelles technologies de l'information et de la communication comme instruments de la pratique professionnelle (numérisation et informatisation des données et des dossiers, administration du travail) entraîne une modification fondamentale du travail : tout acte médical et de soin se doublant d'une saisie de données, l'institution doit désormais faire preuve de " réalisme gestionnaire » (de Gaulejac, 2005). Une nouvelle configuration se dessine, reposant sur l'exigence de relier planification et évaluation par des processus d'adaptation continus. Cependant, la planification n'est possible que lorsque les variables à programmer sont connues à l'avance et mesurables. S'impose ainsi l'exigence de correspondances entre pathologie, légitimité d'une intervention préventive ou curative et compétences des agents médicaux et de soins sollicités. Ainsi, la " rationalité économique » fait appel à la " rationalité médicale » (Good, 1994), aune à laquelle on mesure l'adéquation et le bien-fondé des pratiques préventives et des projets thérapeutiques par des variables scientifiquement fondées. Les objectifs à atteindre deviennent explicites et les étapes à suivre strictement définies par la formalisation de protocoles.

Si ces réformes permettent à plusieurs secteurs de la médecine de s'y référer avec adéquation, une application générale de cette vision peut poser des problèmes, notamment dans le domaine de la santé mentale. De fait, lorsque l'on est confronté à des situations complexes, les réponses soignantes et thérapeutiques requièrent souvent d'être moins systématiques que systémiques. Cette situation soulève une pléthore de questions et conduit à des décisions qui sollicitent simultanément les politiques de santé, l'organisation institutionnelle et les pratiques professionnelles. Ces différents registres définissent les contours de ce qu'est la santé publique tout en constituant les niveaux de son action.

Alterstice-Revue Internationale de la Recherche Interculturelle, vol. 4, $n^{\circ} 2$ 


\section{Transitions de la santé mentale, modification de la relation de soin}

Plus spécifiquement, la santé publique se réfère à « un domaine d'activités qui porte sur le bien-être des collectivités, ou du moins sur la prévention des maladies et peut-être la prolongation de la vie " (Fassin et Hauvray, 2010, p. 7). Elle se situe à l'interface des politiques de gouvernance et des disciplines scientifiques, notamment l'épidémiologie, la médecine, l'économie, la communication et les sciences sociales. Elle élabore ses concepts et ses théories, s'institutionnalise au niveau scientifique et universitaire. Son émergence s'accompagne de la naissance d'un ensemble de normes, de valeurs et de savoirs qui traduisent une forme particulière de " gouvernement de la vie ». Cette gouvernance des collectivités, produit d'une longue maturation, trouve " son enracinement dans un réformisme à visée universelle à partir duquel pouvoirs politiques et savoirs scientifiques prétendent œuvrer en tous domaines au progrès de l'humanité » (Dozon et Fassin, 2001, p. 345), notamment par la promotion de la santé et la lutte contre les maladies. En effet, sa singularité tient à ce qu'elle lie « les différentes significations de la vie, le vivant et le vécu, [...], le physique et le social, ou encore le biologique et le biographique » (Fassin et Hauvray, 2010, p. 8). Ces connaissances scientifiques s'intensifient d'un regard inédit par lequel la santé publique " pense en termes de populations et non plus d'individus ": en ce sens, elle constitue une " conquête morale ", promouvant « un double principe de solidarité et de responsabilité, par lequel la société se mandate elle-même pour s'occuper du bien-être collectif » (Fassin et Hauvray B., 2010, p. 9). Sa perspective est donc unique et s'articule à la fois à la réalité épidémiologique, médicale et thérapeutique de la maladie et à la réalité des acteurs, des pratiques et des institutions.

La santé mentale constituant un secteur de la santé publique, elle assure les mêmes missions, partage les mêmes objectifs et utilise les mêmes instruments. De fait, la gestion des troubles mentaux, historiquement réglementée par la psychiatrie, relève désormais de la santé mentale, secteur appelé à organiser, par ses politiques, institutions et professions une réponse à la situation psychologique des individus. Plus particulièrement, ces pratiques incluent des interventions aux niveaux sociétal et médiatique, institutionnel et pédagogique, individuel et thérapeutique, dans le but de prévenir des troubles mentaux avérés, éviter leur détérioration et promouvoir la santé mentale, le bien-être et la qualité de vie (De Piccoli, 2014). Les caractéristiques individuelles relevant de la santé mentale sont, quant à elles, déclinées en plusieurs registres : souffrance psychique, capacité, fonctionnement social, état positif, capital de santé normatif (Lovell, 2010, p. 162). La réalisation de ses objectifs - diminuer la prévalence des troubles mentaux et leur impact néfaste sur la santé et accroître la capacité des individus à faire face aux événements stressants de la vie - participe en même temps d'une mouvance historique caractérisée par un basculement de référentiels concernant la psychiatrie (Ehrenberg et Lovell, 2005; Otero, 2003). Après une phase de «Grand désenfermement " (qui a notamment déplacé la prise en charge de l'individu fragilisé et vulnérable de l’hôpital psychiatrique vers la communauté et la critique de certaines formes de médicalisation), le "Grand renversement » se caractérise par une valorisation de l'usager, qui devient l'acteur individuel de " son " problème de santé mentale et de la résolution possible de ce dernier. Au centre de tout dispositif thérapeutique et de recherche clinique, son consentement éclairé est désormais indispensable (Ehrenberg, 2010). Penser en ces termes l'usager des services de santé mentale va de pair avec la nécessité de repositionner la psychiatrie dans la société. Ainsi, dans les sociétés contemporaines, la notion de santé mentale peut être considérée comme l'expression d'une réorganisation des rapports entre maladie, santé et socialisation (Ehrenberg, 2005).

Cette transformation se construit à travers trois processus d'intégration propres à la psychiatrie, respectivement dans la médecine, dans la santé publique et dans la cité. La première renvoie à un renforcement de l'insertion de la psychiatrie, branche médicale, dans la médecine, avec des répercussions importantes au niveau scientifique et clinique. La valorisation des preuves afin d'établir les décisions les plus appropriées pour la pratique se généralise. Cette évolution vise à formaliser ce qui est scientifiquement "légitime» dans la clinique. S'appuyant sur l'émergence d'une médecine basée sur les preuves (EBM), l'exigence de scientificité en psychiatrie et en psychothérapie apparaît de prime abord comme légitime : d'un point de vue clinique, elle implique l'association de l'expertise établie par le clinicien et les meilleures preuves cliniques du champ de la production scientifique. Ce principe est donc censé assurer une autonomie professionnelle, un jugement clinique et une intégrité thérapeutique. Dans les faits, face à la standardisation croissante des pratiques, un conflit potentiel risque cependant d'émerger entre la singularité du professionnel, les spécificités de chaque relation thérapeutique et l'application de pratiques dont l'efficacité a objectivement fait ses preuves (Amstrong, 2002). 
Ainsi, certains modèles et théories sont valorisés au détriment d'autres, notamment la promotion du paradigme neurocognitifau détriment des méthodes de psychothérapie plus classiques. Preuve en est le modèle de la plasticité cérébrale et ses applications cliniques. Ce nouveau paradigme décrit le cerveau comme un amalgame de mille milliards de neurones, connectés à leur tour par une centaine de substances chimiques qui engendrent des milliards de connexions, l'absence d'une substance ou le dérèglement d'une interaction biochimique pouvant provoquer des symptômes et être à l'origine d'un problème individuel. Tout est une affaire de cerveau, de neurotransmetteurs et de neurorécepteurs, ou encore de niveau de sérotonine. II en découle que la santé psychique s'inscrit dans le biologique, et que les réponses aux problèmes individuels résident dans l'action sur les constituants neurologiques par la prescription de médicaments. Bien que cette logique causale des problèmes psychiques soit scientifiquement fondée, l'appréhension des souffrances humaines par la parole et la relation, qui sont susceptibles de décoder les trajectoires de vie et des les inscrire dans un processus de symbolisation, amène une autre vision et une autre pertinence. Dès lors, ce qui fonde les procédures de légitimation (" les meilleures preuves ", "l'expertise " ou encore "la rationalité ») est soumis aux débats scientifiques, aux conflits d'intérêt et aux enjeux politiques et économiques, et s'avère donc contestable et variable temporellement et géographiquement (Amstrong, 2002). Les débats qui en découlent procèdent en partie de la difficulté à établir un consensus scientifique et thérapeutique sur bon nombre de situations cliniques. II n'en reste pas moins que l'avènement de la preuve en santé mentale redessine la nature, et donc la direction, des pratiques cliniques.

La deuxième intégration relie la psychiatrie à la santé publique, ce qui entraîne des contraintes économiques, soustendues par un cadre légal, et ce qui contraint à penser la clinique aussi en termes d'efficience et de coûts sociaux. La création et la formalisation de procédures standardisées, tant au niveau de l'évaluation diagnostique que de la prise en charge thérapeutique, et l'existence de protocoles adaptés à chaque pathologie, des "guidelines" susceptibles de tracer les étapes des interventions, deviennent la norme. Cette tendance ajoute aux propriétés qualitatives d'une relation de soin basée sur les dimensions intersubjective et relationnelle - notamment la proximité et la durée qui permettent de construire une relation de soin de qualité, la complexité et la spécificité de la demande, les modalités de coopération et de collaboration entre professionnels du secteur - une démarche axée sur la formalisation des actes de soins, leur calibrage et leur reproductibilité (Demailly, 2008). Cette nouvelle complémentarité conduit à un fractionnement des pratiques professionnelles, à l'interchangeabilité entre soignants et à la prééminence de l'acte de soins sur la relation de soin. Le processus en est connu : la démarche quantitative partage le " faire » de chaque profession en une série d'opérations partielles, ayant chacune sa finalité et sa spécificité. Ce qui fonde le management sanitaire, c'est ainsi "la métaphore de l'organisation machine » (Zulueta, 2013), toute référence clinique devant être compatible avec le " modèle machiniste ", fait de procédures et de monitorages, sur la base de catégories diagnostiques de type DSM ou, en Suisse par exemple, DRG/TARPSY (rétribution financière par diagnostic). Ainsi, la réforme des systèmes de santé est destinée à produire des indicateurs statistiques susceptibles de garantir le contrôle gestionnaire et donc les prérogatives économiques du système. II en ressort une philosophie dans laquelle on soigne des troubles plutôt que des individus souffrants.

Cette tendance se généralise et tend à modifier le statut des soignants : au modèle du professionnel apte à faire le choix d'actions pertinentes sur la base de son expérience et de son jugement clinique on préfère le modèle de compétences pré-établies qui dicte les actions du soignant. Cette logique de prescription repose sur des objectifs précis: soigner des pathologies signifie définir a priori - au nom de l'efficience - les éléments du projet thérapeutique, les interventions et leur organisation, les médicaments à prescrire ainsi que la durée du traitement. Cette organisation du travail tend à transformer les professionnels en exécutants de cette standardisation. Tout acte clinique viserait ainsi moins la consolidation d'une relation de soin que l'application de prescriptions : à l'agir reposant sur un choix motivé par une finalité éclairée se substitue le faire comme production de résultats (Galimberti, 2008; Rossi, 2002 et 2011). Alors que l'agir relève de la compétence du professionnel et de son jugement clinique, la logique du faire le contraint à user de recommandations. Si le premier terme implique une action tendue vers un but, le second se limite à la réalisation d'une tâche.

Dès lors, la relation entre travail professionnel et responsabilité personnelle se reconfigure. Toute perspective clinique étant soumise au calcul rationnel de l'appareil technique et scientifique, les professionnels qui opèrent comme des "techniciens " dans ce système ne peuvent désormais plus le modifier, mais seulement le perpétuer (Rossi, 2002). Certes, cette formulation est radicale, mais elle pose une question légitime : si ce sont le rôle et la 
fonction de prestataires de soins qui sont déterminants, le domaine de la santé mentale se réduit-il à la gestion des actions, des interactions et des structures? L'introduction d'une culture managériale au sein de la clinique se révèle alors comme une entrave potentielle aux décisions éclairées des soignants.

La troisième intégration inscrit la santé mentale dans la cité, ce qui modifie le contexte des pratiques. Elle favorise en effet le passage d'une logique d'expertise professionnelle et institutionnelle spécifique à la psychiatrie à une logique de santé collective et publique. Cette situation amène les institutions psychiatriques à inscrire leurs pratiques dans un réseau territorial de santé mentale, ce qui conduit à un essor de la collaboration interprofessionnelle et interinstitutionnelle. Le travail en réseau réforme la coordination entre disciplines et la coopération entre professions et domaines professionnels, notamment en santé, en éducation, dans le domaine social, en droit. Il favorise une prise de conscience majeure, à savoir que toute réponse clinique et thérapeutique à des expériences de fragilité psychique ne peut être dissociée de la portée sociale qu'elle implique. Plus particulièrement, les réseaux travaillent sur le fragile équilibre entre autonomisation croissante des individus, incités à devenir acteurs de leur santé, et exercice d'un contrôle social visant à influencer les comportements et les pratiques en matière de santé (Demailly, 2008).

Si la clinique du suivi et de l'accompagnement en réseau demeure un effort professionnel pour les soignants, elle constitue en même temps une nécessité impérative du social. De fait, avec l'émergence du sujet et de son autonomie, ce n'est pas seulement " la maladie et la pathologie qui changent, mais aussi la santé et la normalité " (Ehrenberg, 2005, p. 19). II s'ensuit que la finalité clinique n'est plus seulement de "soigner une pathologie » mais de permettre à un individu fragilisé de se réinserer dans son environnement social, professionnel et familial. Les interventions "psy " (psycho-sociales, psycho-thérapeutiques, psycho-éducatives, psychiatriques, psychopharmacologiques) contribuent au rétablissement de l'autonomie des personnes afin de palier les "dysfonctionnements", autrement dit rendre les attitudes et les comportements plus " adaptés ». Ces pratiques oscillent entre la socialisation, le dressage et la thérapeutique (Otero, 2005), ne se référant plus exclusivement aux interfaces entre normal et pathologique, mais valorisant davantage le couple " adapté »/« inadapté », en réponse et en écho aux structures sociales et aux règles de socialisation (Ehrenberg et Lovell, 2005). Le rapport entre soin et autonomie devient ainsi central en santé mentale et constitue désormais la pierre angulaire non seulement du traitement mais aussi de la demande. Avec l'autonomie, en effet, nous assistons à une "psychologisation croissante de différents phénomènes et épisodes de la vie des sujets [...] par le biais de leur association directe au domaine de la santé mentale, de la souffrance psychique et du psychologique » (Otero, 2005, p. 6). Ce qui nous amène à questionner le sens de la relation, puisque "le point très délicat pour trouver un accord possible définissant, ou même seulement délimitant, ce qui serait un état de bonne santé mentale, est en partie contourné si l'on prend pour point de départ non plus le fonctionnement psychique du sujet, mais son comportement, c'est-àdire ce qui est observable et objectivable, et par là même soumis aux lois du fonctionnement social " (Bourdillon, 2011, p. 183). L'intégration de la psychiatrie dans la cité tend à modifier la finalité même des interventions cliniques.

\section{Diversité et santé mentale : convergence et antagonismes}

Domaine multidimensionnel aux interactions complexes, la notion de santé mentale se construit par et avec la société. Les transitions économiques, politiques et culturelles qui la traversent ont de profondes incidences sur les perspectives cliniques et le statut des professionnels. Cette situation nous permet de comprendre pourquoi la clinique est non seulement un espace soignant et interpersonnel, mais aussi un espace social traversé par un ensemble de normes et de valeurs, de modèles sociaux et professionnels, d'implications politiques et économiques (Fortin et Laprise, 2007). Ainsi, le travail d'appréhension de la diversité est soumis à une double injonction. La première soulève un défi interne, caractérisé par l'exigence d'une clinique capable de s'adapter à la spécificité et de respecter la singularité de chacun et la seconde représente un défi externe, déterminé par des logiques d'efficience, de planification, de gestion et de standardisation.

La première injonction amène à repenser les modalités du relationnel. Tout d'abord parce que chaque personne est médiatrice de diversité, comprise comme une historicité, une subjectivité et une corporéité spécifiques. Elle est en même temps incitée à devenir actrice de sa santé, en faisant appel à ses ressources et à ses relations. Face à la 
crise psychosociale et aux psychopathologies, souvent chroniques, des individus, les professionnels doivent répondre à des demandes de plus en plus complexes. La clinique du suivi et de l'accompagnement doit permettre de coordonner des rôles, de clarifier des stratégies, de promouvoir un travail de santé en réseau. Cette approche socialisée de la clinique invite les professionnels à réinventer des modes de travail dans un esprit interdisciplinaire et interprofessionnel, à trouver du temps, à garantir de la souplesse pour atteindre des solutions ajustées aux besoins individuels des enfants, des jeunes et des adultes en difficulté. C'est pourquoi la relation de soin se distingue de son ancrage prescriptif, puisqu'elle traite des êtres singuliers dont elle cherche à améliorer l'état de santé. Elle repose sur la communication, dans laquelle des éléments spécifiques entrent en jeu : représentations, normes, valeurs, loyautés, alliances, interactions, émotions, langages. La singularité du professionnel, sa subjectivité, ses prédispositions sont ici très importantes (Vannotti, 2006), dans la mesure où la pratique en santé mentale ne peut pas être réduite à ce qui doit être fait, mais elle " est toujours, dans le même temps, un choix et une décision entre des possibilités »(Gadamer, 1998). Ainsi, une pratique clinique tenant compte de la diversité implique une intelligence dialectique, à la fois analytique et synthétique. Elle présuppose une activité à la fois théorique et pratique et elle véhicule une rationalité qui ne peut se décliner qu'au pluriel, impliquant plusieurs démarches, aussi bien scientifiques que réflexives: connaissances, interactions, décisions et traitements. La clinique contribue à construire l'humain : elle garantit l'avènement continuel de la singularité, parce qu'elle permet de défendre la personne dans son irréductibilité, sa singularité et sa diversité. Et elle doit garantir de pouvoir continuer à le faire : c'est là le premier défi auquel elle doit faire face.

De nos jours, cette diversité ne peut pourtant se penser qu'en relation à une "culture " de l'efficience, de la planification, de la standardisation et de la gestion. La deuxième injonction concerne ainsi une réorganisation institutionnelle importante, sous l'influence de facteurs économiques et politiques. Cette tendance est générale et se définit par une série de phénomènes transnationaux et globaux, au cœur desquelles la santé mentale prend une place importante : il s'agit notamment de l'émergence d'une santé globale, de l'efficience des systèmes, de la gestion des pathologies, de la mondialisation des activités des firmes pharmaceutiques, de la mobilité des savoirs scientifiques et de la circulations des professionnels de la santé. Bien évidemment, cet enjeu global de la santé ne peut se comprendre qu'à l'aune d'un contexte plus local, dans lequel de nombreux éléments sociologiques, culturels, politiques mais aussi épidémiologiques et institutionnels favorisent ou entravent ces stratégies et ajustements. Ces phénomènes transforment la volonté de maîtrise et d'anticipation de la maladie, de ses effets ainsi que des coûts assumés par la collectivité pour y parvenir en une norme politique collective (Hours, 2001). Cette norme de santé tend à affaiblir les spécificités d'une relation de soin en faveur d'une vision transversale et rationnelle de la prise en charge des pathologies. L'asymétrie qui en découle peut avoir des conséquences sur le statut des professionnels et sur la position des sujets souffrants. Pour les soignants, cela se traduit par une logique du travail qui vise à renforcer leurs compétences par une rationalisation de leurs pratiques. Soumis à des logiques qui tendent à transformer tout sujet, et donc toute diversité, en un trouble rationalisé et normalisé, les soignants participent de facto à un projet d'optimisation où le sujet est nié (Illich, 2004). Ainsi, favoriser l' " adéquation » des usagers aux stratégies de l'efficience redéfinit non seulement le statut des professionnels mais aussi le statut et la place de l'être humain souffrant. Appelés à devenir des prestataires de soins de "cas" ou de troubles, les professionnels risquent de perdre leur culture de la complexité et de l'incertitude au profit d'une conduite d'efficience chiffrée : ils font davantage l'objet de contrôles et doivent rendre des comptes, ce qui les incite souvent à prescrire des traitements plus courts (Zulueta, 2013). Le risque de privilégier l'aspect gestionnaire au détriment d'une relation de soin est un défi réel.

Au sein de la santé mentale, la promotion d'une clinique de la diversité, louable par les finalités qu'elle poursuit, tend à se complexifier à l'épreuve des faits. Le facteur humain - le plus impondérable, le plus aléatoire, le moins rationnel -, s'il reste le fondement dans la mise en œuvre d'une relation, demeure en effet le moins quantifiable. $C^{\prime}$ est en ce sens que toute relation de soin constitue l'avènement continuel de la singularité, qui est censée défendre la personne dans son irréductibilité (Bibeau, 2010). Mais ce sont précisément ces formes-là de la diversité et de la singularité qui tendent aujourd'hui à s'effacer. Ces considérations demandent bien sûr à être nuancées et adaptées (Waytz, 2013) : les soignants sont susceptibles de libérer des ressources cognitives aptes à résoudre des problèmes critiques, tout comme ils sont susceptibles de reproduire parfois le conformisme gestionnaire et de faire preuve d'un manque d'esprit critique.

Alterstice - Revue Internationale de la Recherche Interculturelle, vol. 4, $n^{\circ} 2$ 
Dans sa relation à la santé mentale, la diversité devient alors, par analogie, une « condition cosmopolite » (Agier, 2013), une figure à double face : d'un côté, émerge un individu absolu, universel et anonyme, de l'autre, un sujet, qui revendique sa place et son existence sociale tout en reconnaissant celle des autres. Comment concilier ces deux tendances, qui mettent les professionnels dans une posture parfois paradoxale? Promouvoir une réflexivité, voire une analyse, de ces enjeux participe sans doute à transcender les antagonismes propres à la diversité, et contribue ainsi à faire le lien entre personne souffrante et gestion de son « cas».

\section{Bibliographie}

Agier, M. (2013). La condition cosmopolite. L'anthropologie à l'épreuve du piège identitaire. Paris : La Découverte.

Armstrong, D. (2002). Clinical autonomy, individual and collective: the problem of changing doctors 'behaviour'. Social Science and Medicine, 55(10), 1771-1777.

Bibeau, G. (2010). Quel humanisme pour notre âge bio-technologique?, Anthropologie et santé [en ligne], 1|2010. http://anthropologiesante.revues.org/91

Bourdillon, F. (2011). Prévention et santé mentale de l'enfant : les questions éthiques soulevées par des approches ciblées, Santé publique, 23, 181-188.

Castro, A. et Singer, M. (dir.). (2004). Unhealthy health policy: A critical anthropological examination. Altamira Press.

Cognet, M. et Montgomery, C. (2007). Éthique de l'altérité. La question de la culture dans le champ de la santé et des services sociaux. Québec : Presses de l'Université Laval.

Demailly, L. (2008). Politiques de la relation. Approche sociologique des métiers et activités professionnelles relationnelles. Villeneuve d'Ascq, France : Presses universitaires du Septentrion.

Demezeux, S. (2013). Qu'est-ce que le DSM? Genèse et transformations de la bible américaine de la psychiatrie. Paris : Éd. d'Ithaque.

De Piccoli, N. (2014). Salute e qualità della vita nella società del benessere. Rome : Carrocci Editore.

Domenig, D. (2007). Transkulturelle Kompetenz. Berne : Hans Huber.

Dozon, J.-P. et Fassin, D. (dir). (2001). Critique de la santé publique. Une approche anthropologique. Paris : Balland.

Ehrenberg, A., (2005). La plainte sans fin. Réflexions sur le couple souffrance psychique/santé mentale. Cahiers de recherche sociologique, 41-42, 17-41.

Ehrenberg, A. (2010). La société du malaise. Paris : Odile Jacob.

Ehrenberg, A. et Lovell, A. (dir.). (2005). La maladie mentale en mutation : psychiatrie et société. Paris : Odile Jacob.

Fassin, D. et Hauray, B. (2010). Santé publique. L'état des savoirs. Paris : La Découverte.

Fortin, S. et Laprise E. (2007). L'espace clinique comme espace social : culture, compétence, dynamique relationnelle. Dans M. Cognet et C. Montgomery (dir.), Les représentations de l'Autre dans l'intervention : pour une éthique de l'altérité (p. 191-214). Québec: Presses de l’Université Laval.

Gadamer, H.-G. (1998). Philosophie de la santé. Paris : Grasset et Mollat.

Galimberti, U. (2008). La morte dell'agire e il primato del fare nell'età della tecnica. Milan : Albo Versorio.

Garcia Canclini, A. (coord.). (2011). Conflictos interculturales. Barcelone : Gedisa Editorial.

de Gaulejac, V. (2005). La société malade de la gestion. Idéologie gestionnaire, pouvoir managériale et harcèlement social. Paris : Seuil

de Gaulejac, V. (2009). Qui est Je? Paris : Seuil.

Alterstice - Revue Internationale de la Recherche Interculturelle, vol. $4, n^{\circ} 2$ 
Goguikian Ratcliff, B. et Strasser, O. (dir.). (2009). Clinique de l'exil : chroniques d'une pratique engagée. Genève : Georg Éditeur.

Good, B. (1994). Medicine, rationality and experience. An anthropological Perspective. Cambridge : University Press.

Gori, R. et Del Volgo, M.-J. (2008). Exilés de l'intime. La médecine et la psychiatrie au service du nouvel ordre économique. Paris : Denoël.

Hours, B. (dir.). (2001). Systèmes et politiques de santé : de la santé publique à l'anthropologie. Paris : Karthala.

Illich, I. (2004). CEuvres complètes. Paris : Fayard.

Kehr, J., Niewöhner, J. et Vailly, J. (2011). De la vie biologique à la vie sociale. Approches sociologiques et anthropologiques. Paris : La Découverte.

Kleinmann, A. (1995). Writing at the margin. Discourse between anthropology and medicine. Berkeley : University of California Press.

Littlewood, R. (2002). Pathologies of West. An anthropology of mental ilness in Europe and America. Ontario : Cornell University Press.

Marzano, M. (dir.). (2007). Dictionnaire du corps. Paris : Presses universitaires de France.

Nathan, T. (1994). L'influence qui guérit. Paris : Odile Jacob.

Otero, M. (2003). Les règles de l'individualité contemporaine. Santé mentale et société. Québec : Presses de l’Université Laval.

Rossi, I. (2002). Réseaux de santé, réseaux de soins. Culture prométhéenne ou liberté de l'impuissance? Tsantsa, 7, $12-21$.

Rossi, I. (2011). Réseaux, coopérations interprofessionnelles et cultures politiques de la santé. Collectif : Le travail d'équipe en PMI. Comment penser/panser ensemble? (p. 17-26). Paris : Syndicat national des médecins de protection maternelle et infantile (SNMPMI).

Rossi, I. (2012). Sociétés plurielles pour les générations futures. Dans E. Corbaz (dir.), Pages d'accueil (p. 19-25). Lausanne : Éditions Antipodes.

Rossi, I. (2013). Identités et travail. La preuve par le corps. Dans B. Danuser et V. Gonik (dir.), Der Arbeit : eine Revision. Le travail : une re-vision (p. 257-269). Zürich : Chronos Verlag.

Sabatier, C. et Douville, O. (2002). Culture et santé mentale : quels liens? Pour une approche complexe et plurielle. Dans C. Sabatier et O. Douville (dir.), Cultures, insertions et santé (p. 7-30). Paris : L’Harmattan.

Selot, S. (2001). Relations interethniques et classifications : de l'emploi du diagnostic dans la littérature psychiatrique du XX $X^{\mathrm{e}}$ siècle. L'Autre. Cliniques, cultures et sociétés, 2(3), 481-494.

Tison, B. (2007). Soins et cultures. Formation des soignants à l'approche interculturelle. Issy-les-Moulineaux : Elsevier/Masson.

Touraine, A. (2005). Un nouveau paradigme. Pour comprendre le monde d'aujourd'hui. Paris : Fayard.

Vannotti, M. (2006). Le métier de médecin : entre utopie et désenchantement. Genève : Médecine et Hygiène.

Waytz, A. (2012). Dehumanization in medicine. Causes, solutions and fonctions. Perpsectives on Psychological Science, 7, 176-186.

Welsch, W. (2005). Trankulturelle Gesellschaften. Dans P.-U. Merz-Benz et G. Wagner (dir.), Kultur in Zeiten der Globalisierung. Neue Aspekte einer soziologischen Kategorie (p. 39-67). Frankfurt-am-Main : Humanities Online.

Wolton, D. (2008). "Conclusion générale : de la diversité à la cohabitation culturelle ", Hermès, La Revue, 51, 195-204.

Zulueta, P. (2013). Compassion in $21^{\text {st }}$ century medecine: is it sustainable ? Journal of Clinical Ethics, 8, 119-128.

Alterstice - Revue Internationale de la Recherche Interculturelle, vol. 4, $n^{\circ} 2$ 